\title{
ACL graft re-rupture after double-bundle reconstruction: factors that influence the intra-articular pattern of injury
}

\author{
Carola F. van Eck • Eric J. Kropf • James R. Romanowski • Bryson P. Lesniak • \\ Michael J. Tranovich • C. Niek van Dijk • Freddie H. Fu
}

Received: 16 June 2010/Accepted: 11 October 2010/Published online: 18 November 2010

(C) The Author(s) 2010. This article is published with open access at Springerlink.com

\begin{abstract}
Purpose To determine the most common rupture patterns of previously reconstructed DB-ACL cases, seen at the time of revision surgery, and to determine the influence of age, gender, time between the initial ACL reconstruction and re-injury, tunnel angle and etiology of failure.

Methods Forty patients who presented for revision surgery after previous double-bundle ACL reconstruction were enrolled. Three orthopedic surgeons independently reviewed the arthroscopic videos and determined the rupture pattern of both the anteromedial and posterolateral grafts. The graft rupture pattern was then correlated with the previously mentioned factors.

Results The most common injury pattern seen at the time of revision ACL surgery was mid-substance AM and PL bundle rupture. Factors that influenced the rupture pattern (proximal vs. mid-substance and distal rupture vs. elongated, but in continuity) were months between ACL reconstruction and re-injury $(P=0.002)$, the etiology of failure (traumatic vs. atraumatic) $(P=0.025)$ and the measured graft tunnel angle $(P=0.048)$.

Conclusions The most common pattern of graft re-rupture was mid-substance AM and mid-substance PL. As the
\end{abstract}

C. F. van Eck · E. J. Kropf · J. R. Romanowski ·

B. P. Lesniak · M. J. Tranovich · F. H. Fu ( $\square)$

Department of Orthopaedic Surgery,

University of Pittsburgh Medical Center,

Kaufman Building suite 1011, 3471 Fifth Avenue,

Pittsburgh, PA 15213, USA

e-mail: ffu@upmc.edu

C. F. van Eck · C. N. van Dijk

Orthopaedic Research Centre Amsterdam,

Department of Orthopaedic Surgery, University of Amsterdam,

Academic Medical Center, Meibergdreef 9,

1100 DD Amsterdam, The Netherlands length of time from the initial DB-ACL reconstruction to revision surgery increased, the pattern of injury more closely resembled that of the native ACL. Evaluation of patients who have undergone double-bundle ACL reconstruction, with a particular focus on graft maturity, mechanism of injury and femoral tunnel angles, and graft rupture pattern assists in preoperative planning for revision surgery.

Keywords Anterior cruciate ligament - Double-bundle ACL $\cdot$ Revision surgery · Rupture pattern · Graft failure

\section{Introduction}

The exact etiology of anterior cruciate ligament (ACL) injuries remains poorly understood. Although the role of the two functional bundles (anteromedial (AM) and posterolateral (PL)) has been defined, few studies exist that attempt to establish the exact pattern or patterns of injury that may occur [26]. Similarly, specific factors that can influence the relative pattern of injury are yet to be defined.

Biomechanical studies have shown that variations in ACL tunnel placement result in variable postoperative knee kinematics and loads [2, 6, 16, 21, 22, 25]. Schenck et al. employed a straight knee hyperextension model to rupture the native ACL and demonstrated a variable pattern of ACL tears at high vs. low energy moments [11]. This suggests that there are multiple biomechanical factors that can influence rupture pattern.

Recently, Zantop et al. [26] studied the rupture pattern of the native ACL at the time of arthroscopy. Their results demonstrated that $24 \%$ of patients had a partial ACL rupture and in $44 \%$ of the total cases, the AM and PL bundle did not rupture at the same level (proximal, mid-substance, distal). 
With the increasing number of ACL reconstructions being performed annually, a subsequent increase in the number of revision cases has occurred [12, 18]. Recently, greater attention is being paid to re-rupture cases and what can be learned in this setting $[4,13]$. Biomechanical studies [7, 16, 21-24] have shown that variations in ACL reconstruction technique, i.e. difference in tunnel placement and graft type, result in variable postoperative knee kinematics and loads. Theoretically, this exposes the reconstructed ACL graft to variable forces and potentially results in different rupture patterns when the graft is stressed to failure. To date, no studies have specifically attempted to determine the most common rupture pattern and causative or related factors in failure of double-bundle ACL reconstruction. Double-bundle ACL reconstruction may have additional causes of failure than single-bundle reconstruction, associated with 4 tunnels instead of 2 . If causes of failure can be determined, as well as factors that can identify these causes, they can be prevented in the future.

The primary purpose of this study was to determine the most common rupture pattern in ACL revision cases after previous double-bundle reconstruction. Our secondary purpose was to identify variables that can be correlated with rupture pattern of the graft. The hypothesis of this study was that it is possible to identify factors that influence the rupture pattern of the ACL graft, such as the time between the primary surgery and the failure and the mechanism of injury.

\section{Materials and methods}

Between 2005 and 2009, all patients who underwent revision ACL surgery after a previous double-bundle reconstruction were evaluated. After receiving approval from our institutional review board, forty patients were identified and prospectively enrolled as a cohort. We excluded all patients who had undergone multiple $(>1)$ revision surgeries on the knee of interest.

Three orthopedic surgeons with sports medicine fellowship training independently reviewed the arthroscopic footage of the included subjects to determine the rupture pattern. The rupture pattern was recorded using the classification system previously described by Zantop et al.[26] Specifically, each bundle was classified independently as ruptured proximally, mid-substance or distally, functionally insufficient due to elongation, or intact/ uninjured. The observers were not blinded to information about the previous reconstructive procedure. If the observers felt they were unable to determine the rupture pattern due to insufficient footage, they could score a bundle as indeterminate. Inter-observer agreement was also determined.
Patient demographic data including age, gender, date of the initial ACL reconstruction, date of the re-injury and etiology of failure were collected. The etiology of failure was classified as either traumatic or atraumatic.

Finally, the femoral graft tunnel angles were measured on standardized anterior-posterior radiographs of the knee, using a commercially available radiographic imaging system (Stentor, Philips Healthcare, Andover, MA). The tunnel angle was defined as the angle between the tunnel and the axis of the femur (Fig. 1).

\section{Statistical analysis}

Data were analyzed with PASW Statistics (version 17.0, SPSS Inc., Chicago, IL). Inter-observer agreement for the three observers was calculated using the Fleiss kappa coefficient for multiple ratings per subject. This method generates a single kappa value for all three observers combined.

Statistical analysis of associated variables was performed using the rupture pattern ratings from observer number one. Descriptive statistics were calculated for all the variables, including frequency counts for nominal variables and means, medians, standard deviation and a range for all continuous variables.

The Kruskal-Wallis one-way analysis of variance was used to determine the influence of patient age, ACL reconstruction to re-injury time interval and tunnel angle on the rupture pattern. When statistical significance was

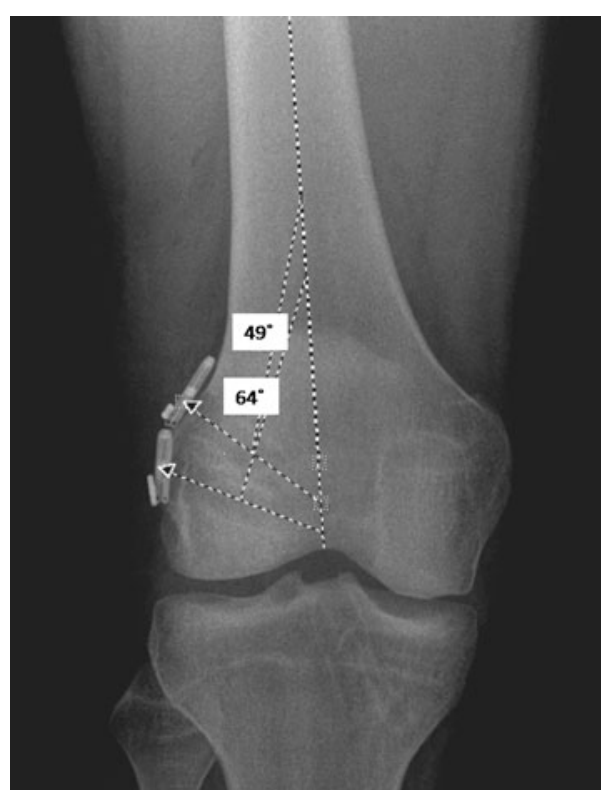

Fig. 1 Anterior-posterior flexion weight-baring radiograph of the right knee after double-bundle ACL reconstruction. The tunnel angle is measured as the angle between the axis of the femur and the outline of the tunnel 
achieved $(P<0.05)$, a post-hoc Mann-Whitney $U$ test was performed to further specify the difference. For the posthoc analysis, the Bonferroni correction was applied to adjust the a priori alpha level to the number of comparisons performed. This resulted in $P<0.05 / 3$ for the AM bundle and $0.05 / 6$ for the PL bundle as the level of significance. The Chi-square test and Cramer V correlation coefficient were used to measure the relationship between the rupture pattern and nominal variables such as gender and etiology of failure. The a priori alpha level was $P<0.05$. When statistical significance was achieved, a post-hoc analysis of the standard residuals was performed. A critical value of \pm 1.96 was used to further specify the difference.

\section{Results}

Forty subjects were included in this study and arthroscopic video reviewed by all examiners. The kappa for multiple observers was .80 for the AM bundle and .76 for the PL bundle.

The demographic information of the study cohort is reported in Table 1. The frequencies of the different rupture patterns are displayed in Table 2. The most frequent rupture pattern was mid-substance rupture of both the AM and PL bundle graft $(35.1 \%)$. After combined mid-substance injuries, the three most common patterns seen were mid-substance rupture of the AM graft with either elongation $(13.5 \%)$ or proximal rupture $(8.1 \%)$ of the PL graft and proximal rupture of the AM bundle with an intact PL bundle (8.1\%) (Fig. 2). Since there was an extremely low number of distal (tibial-sided) graft ruptures, distal and mid-substance ruptures were collapsed as a single category for further statistical analysis.

Isolated analysis of the AM bundle graft showed that the time between the initial ACL surgery and re-injury significantly influenced the rupture pattern. The reconstruction to re-rupture interval was significantly greater in proximal ruptures when compared to distal or mid-substance ruptures (32 vs. 14 months, $P=0.001$ ). Rupture pattern of the AM graft was also significantly correlated with the etiology of failure (traumatic vs. atraumatic) (Cramer $\mathrm{V}=0.436$; $P=0.025$ ). Ninety-four percent of proximal, mid-substance or distal tears were traumatic, while only fifty-seven percent of elongated grafts were the result of a traumatic event. The Kruskall-Wallis test also showed a main effect of tunnel angle on the AM graft rupture pattern, however, post-hoc analysis was unable to further specify this effect. The AM rupture pattern was not significantly different based on the patients' age or gender (Table 3).

With respect to the PL bundle, the time interval between the initial ACL surgery and re-injury also influenced the rupture pattern. Distal or mid-substance ruptures occurred at a significantly shorter reconstruction to re-rupture interval than the proximal ruptures (13 vs. 24 months, $P=0.006)$. The PL rupture pattern was not significantly different based on the patients' age, gender, etiology of failure or tunnel angle $(P=0.054)$ (Table 4).
Table 1 Demographic data $(N=40)$

$S D$ standard deviation, $A M$ anteromedial bundle graft, $P L$ posterolateral bundle graft

Table 2 Frequencies (\% of total) of the different rupture patterns for the AM and PL bundle graft

$P L$ bundle graft,

$A M$ anteromedial bundle, $P L$ posterolateral bundle

\begin{tabular}{|c|c|c|c|c|}
\hline & Ratio & Mean & SD & Range \\
\hline \multicolumn{5}{|l|}{ Gender } \\
\hline Male:Female & $22: 17$ & & & \\
\hline Age & & 19 (median) & & $16-38$ \\
\hline Months between initial surgery and re-injury & & 18.6 & 9.5 & $6-39$ \\
\hline \multicolumn{5}{|l|}{ Etiology of failure } \\
\hline Traumatic:A-traumatic & $35: 5$ & & & \\
\hline Tunnel angle AM & & $23^{\circ}$ & $13^{\circ}$ & $1^{\circ}-41^{\circ}$ \\
\hline Tunnel angle PL & & $43^{\circ}$ & $8^{\circ}$ & $27^{\circ}-62^{\circ}$ \\
\hline
\end{tabular}

\begin{tabular}{lcclccr}
\hline PL & Proximal & Mid-substance & Distal & Elongated & Intact & Total AM \\
\hline AM & & & & & & \\
Proximal & 5 & 0 & 0 & 5 & 8 & 19 \\
Mid-substance & 8 & 35 & 0 & 14 & 3 & 59 \\
Distal & 0 & 3 & 3 & 0 & 3 & 8 \\
Elongated & 5 & 3 & 0 & 0 & 5 & 14 \\
Intact & 0 & 0 & 0 & 0 & 0 & 0 \\
Total PL & 19 & 41 & 3 & 19 & 19 & 100 \\
\hline
\end{tabular}


Fig. 2 Arthroscopic figures of the right knee in $90^{\circ}$ of flexion displaying the four most frequent double-bundle graft rupture patterns after anterior cruciate ligament reconstruction. Together they account for $65 \%$ of the ruptures. a Anteromedial portal view, the $A M$ and $P L$ bundle have ruptured mid-substance.

b Anterolateral portal view, the $A M$ bundle ruptured midsubstance and the $P L$ bundle is elongated. c Anteromedial portal view, the $A M$ bundle ruptured mid-substance and the $P L$ bundle proximal.

d Anteromedial portal view, the $A M$ bundle ruptured proximal and the $P L$ bundle is still intact. $A M$ anteromedial, $P L$ posterolateral, $L F C$ lateral femoral condyle
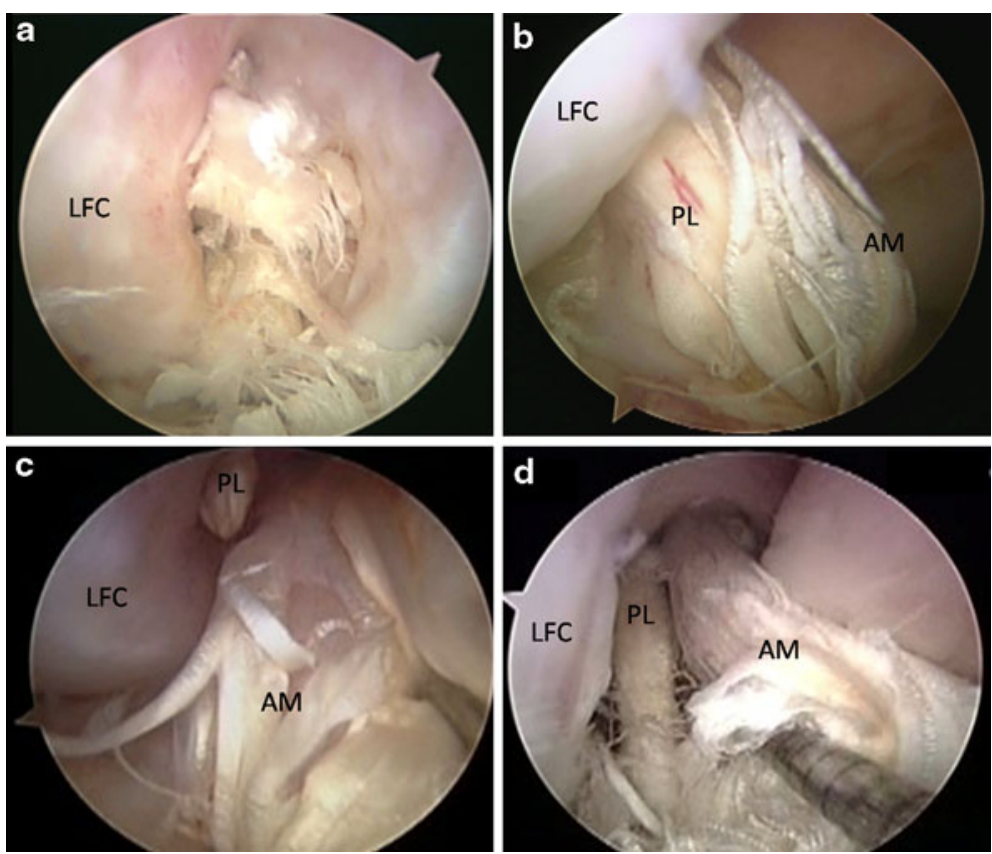

Table 3 Factors that influence the rupture pattern of the anteromedial bundle

\begin{tabular}{|c|c|c|c|c|}
\hline Factors & Proximal $(n=7)$ & Mid-substance and distal $(n=25)$ & Elongated $(n=7)$ & $P$ value \\
\hline Age (median, $[95 \% \mathrm{CI}]$ ) & 20 [18-27] & $18.5[19-21]$ & $23.5[16-34]$ & NS \\
\hline Gender ( $\%$ male $)$ & $57 \%$ & $56 \%$ & $50 \%$ & NS \\
\hline Months out (median, $[95 \% \mathrm{CI}]$ ) & 32 [21-37] & $14[12-18]$ & $17[14-31]$ & $0.002^{\mathrm{a}}$ \\
\hline Etiology of failure (\% traumatic) & $86 \%$ & $96 \%$ & $57 \%$ & $0.025^{\mathrm{b}}$ \\
\hline Tunnel angle (median, $[95 \% \mathrm{CI}]$ ) & $14[8-20]$ & $29[21-31]$ & $20[11-30]$ & $0.048^{\mathrm{c}}$ \\
\hline
\end{tabular}

95\% CI 95\% confidence interval, NS not significant

${ }^{a}$ Proximal ruptures had a significantly greater time between the primary surgery and re-injury than the mid-substance and distal ruptures, $P=0.001$

${ }^{\mathrm{b}}$ Cramer $\mathrm{V}=0.436$. Atraumatic injuries were more likely to be elongated

c Post-hoc analysis was unable to further specify this difference

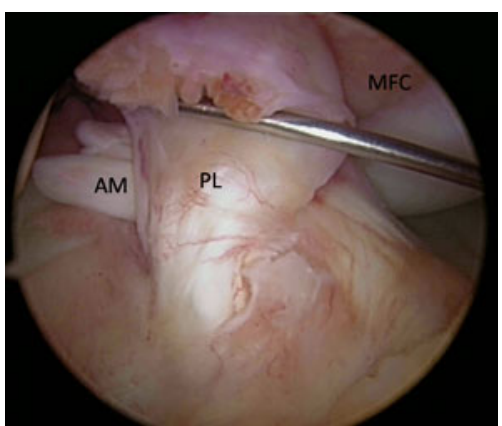

Fig. 3 Arthroscopic figure of the right knee in $90^{\circ}$ of flexion showing the most frequent rupture pattern of the native anterior cruciate ligament. Both the $A M$ and $P L$ bundle are rupture proximally. When compared to the most common rupture pattern in revision surgery (Fig. 2a), it clearly looks different. Anterolateral portal view. AM anteromedial, $P L$ posterolateral, $L F C$ lateral femoral condyle, $M F C$ medial femoral condyle

\section{Discussion}

The most important finding of the present study was that the most common rupture pattern in ACL revision cases after previous double-bundle reconstruction is mid-substance rupture of the AM bundle with either mid-substance rupture, proximal rupture, or elongation of the PL bundle, as well as an isolated proximal AM bundle rupture with an intact PL graft. These four patterns characterized approximately $65 \%$ of all ruptures. This differs from the most frequent rupture pattern seen in the native $\mathrm{ACL}$, as reported by Zantop et al. [26]. Their study demonstrated proximal rupture of the AM bundle with either proximal or midsubstance rupture of the PL bundle as the most common patterns of injury (Fig. 3). However, despite this difference, we did see similar results for the occurrence of both bundles ruptures at the same level and for the relative 
Table 4 Factors that influence the rupture pattern of the posterolateral bundle

\begin{tabular}{|c|c|c|c|c|c|}
\hline Factors & Proximal $(n=7)$ & Mid-substance and distal $(n=16)$ & Elongated $(n=10)$ & Intact $(n=5)$ & $P$ value \\
\hline Age (median, $[95 \% \mathrm{CI}]$ ) & $21[18-26]$ & $18[18-23]$ & $19.5[18-27]$ & 19 [16-29] & NS \\
\hline Gender ( $\%$ male $)$ & $83 \%$ & $56 \%$ & $40 \%$ & $40 \%$ & NS \\
\hline Months out (median, $[95 \% \mathrm{CI}]$ ) & $24[15-32]$ & 13 [12-29] & $17[12-33]$ & $22[15-21]$ & $0.039^{\mathrm{a}}$ \\
\hline Etiology of failure (\% traumatic) & $86 \%$ & $100 \%$ & $70 \%$ & $100 \%$ & NS \\
\hline Tunnel angle (median, $[95 \% \mathrm{CI}]$ ) & $43[35-50]$ & $45[43-51]$ & $40[33-45]$ & $37[33-45]$ & NS \\
\hline
\end{tabular}

95\% CI 95\% confidence intervall, NS not significant

a The proximal ruptures had a significantly longer time between the primary surgery and re-injury than the mid-substance and distal ruptures, $P=0.006$

Table 5 Comparison of the rupture pattern in ACL revision surgery to the rupture pattern of the native ACL

$A C L$ anterior cruciate ligament, $A M$ anteromedial bundle, $P L$ posterolateral bundle

\begin{tabular}{lll}
\hline Measured data & $\begin{array}{l}\text { Zantop et al. [26] rupture } \\
\text { pattern native ACL }\end{array}$ & $\begin{array}{l}\text { Current study rupture } \\
\text { pattern reconstructed ACL }\end{array}$ \\
\hline $\begin{array}{l}\text { Two most frequent rupture } \\
\text { patterns }\end{array}$ & AM proximal PL proximal & $\begin{array}{l}\text { AM mid-substance PL } \\
\text { mid-substance }\end{array}$ \\
$\begin{array}{l}\text { Cases where both bundles ruptured } \\
\text { at the same location }\end{array}$ & $\begin{array}{l}\text { AM proximal PL mid-substance } \\
\text { AM mid-substance PL elongated } \\
\text { PL bundle intact }\end{array}$ & $12 \%$ \\
\hline
\end{tabular}

incidence of an intact PL bundle (Table 5). In another case series of ACL revision surgeries, Kaz et al. [4] also reported an isolated AM bundle rupture with intact PL bundle among their report of three double-bundle reruptures.

Patient-related factors such as the age and gender showed no correlation with the pattern of rupture. The time interval between the initial ACL reconstruction and the reinjury did significantly influence the rupture pattern. A longer time period was associated with more proximal ruptures of the graft, whereas a shorter time resulted in more distal and mid-substance ruptures. We believe that this finding may be the result of varied degrees of healing or "ligamentization" of the reconstructed ACL graft [20]. Stated another way, when the graft was given more time to heal and remodel, the resultant re-injury pattern was most similar to the native ACL, i.e. proximal rupture of the graft [10]. Ligamentization may also be influenced by graft type (allograft vs. autograft) and procedure (single- vs. doublebundle) [5].

Graft tunnel angle also demonstrated significant effect on rupture pattern. However, post-hoc analysis was unable to specify this difference. We believe that tunnel positioning is a key determinant of the biomechanical function of the knee [3]. Theoretically, variable graft tunnel positioning should result in differences in forces seen by the graft. Therefore, we would expect differences in rupture patterns as tunnel position is changed. Further research is needed to investigate and validate these principles.

Lastly, this study showed a relationship between the rupture pattern and the etiology of failure. When the patient self-reported an actual traumatic event, the graft was more often ruptured. In comparison, the atraumatic failure group more frequently demonstrated elongation of the graft. This could potentially be the result of the tunnel placement during the initial procedure. If the tunnel was placed outside of the native ACL insertion site, this could result in a mal-functioning graft that is exposed to unusual forces and as a result elongates over time.

Limitations of the study were the small sample size of 40 patients. In addition, the rupture pattern was established by review of arthroscopic videos captured at the time of the revision surgery. Therefore, observers were not able to control the arthroscope or to probe the ruptured ligament. Still, despite the lack of tactile data, we did find a good to excellent inter-observer reliability. Since a satisfactory correlation was seen between all observers, we deemed it appropriate to utilize a single observer's ratings for further statistical analysis. In only one case, the AM bundle was scored as indeterminate, while the PL rupture pattern could be established. This subject was excluded for the analysis of the AM bundle and included for the analysis of the PL bundle. Another limitation was the sample size, resulting in only one distal rupture. Statistical analysis of these data 
would have resulted in violation of the statistical assumptions. This dilemma was solved by combining the distal and mid-substance ruptures, so no comparisons could be made between these two classes individually. Finally, the rupture pattern may be determined by multiple of the factors assessed in this study, some of which may be confounding. The reconstruction techniques for revision surgery after previous double-bundle ACL reconstruction are still being developed.

Although the outcome of double-bundle reconstruction is generally good $[8,17]$, with the high incidence of ACL injuries, revisions are to be expected. During revision ACL surgery, the rupture pattern of the graft should be inspected in all cases. The pattern of injury may yield valuable information about the function of the primary ACL graft. However, this step requires careful arthroscopic examination. Other methods of determining the pattern of injury have not proven reliable. Isolated rupture of the AM bundle has been shown to have a greater effect on the anterior drawer sign than on the Lachman test. The opposite is true for PL bundle ruptures, which can also be tested using the pivot shift [9]. Determining the exact rupture pattern with conventional magnetic resonance imaging can be difficult. An isolated PL bundle rupture is more difficult to diagnose because of the oblique course of this bundle taking the ligament out of plane when standard MRI sequences are employed. Three-dimensional reconstruction and 3D magnetic resonance imaging (MRI) can better visualize the rupture pattern preoperatively [15], but this requires specialized views or use of ultra high-field 3 Tesla MRI, which are not typically available in routine clinical practice. At present, the rupture pattern can best be observed and classified during arthroscopic examination. Visualizing the rupture pattern during arthroscopy may present a challenge because the AM bundle overlies the PL bundle when viewed from the standard anterolateral portal [9]. Using the figure of four position or viewing through the anteromedial portal will help in the visualization of the whole ACL and both the tibial and femoral insertion sites [1]. If there is an isolated rupture of the AM or PL graft, augmentation surgery should be considered, similar to primary ACL reconstruction [13, 14]. Each encountered situation during revision surgery requires an individual approach [19].

\section{Conclusion}

This study shows that after double-bundle reconstruction, the most common rupture pattern is the failure of both grafts mid-substance. Factors that influence the rupture pattern are the time interval between ACL reconstruction and re-injury, etiology of failure and graft tunnel angle.
Acknowledgments The authors wish to thank Dr. J.J. Irrgang for his helpful discussion concerning the statistical analysis and interpretation of the data. Our department receives funding from Smith and Nephew to support research related to ACL reconstruction.

Open Access This article is distributed under the terms of the Creative Commons Attribution Noncommercial License which permits any noncommercial use, distribution, and reproduction in any medium, provided the original author(s) and source are credited.

\section{References}

1. Cohen SB, Fu FH (2007) Three-portal technique for anterior cruciate ligament reconstruction: use of a central medial portal. Arthroscopy 23:e321-e325

2. Hoshino Y, Nagamune K, Yagi M, Araki D, Nishimoto K, Kubo S, Minoru D, Kurosaka M, Kuroda R (2009) The effect of intraoperative knee flexion angle on determination of graft location in the anatomic double-bundle anterior cruciate ligament reconstruction. Knee Surg Sports Traumatol Arthrosc 17:1052-1060

3. Jepsen CF, Lundberg-Jensen AK, Faunoe P (2007) Does the position of the femoral tunnel affect the laxity or clinical outcome of the anterior cruciate ligament-reconstructed knee? A clinical, prospective, randomized, double-blind study. Arthroscopy 23:1326-1333

4. Kaz R, Starman JS, Fu FH (2007) Anatomic double-bundle anterior cruciate ligament reconstruction revision surgery. Arthroscopy 23:1250.e1-3

5. Kim SJ, Jo SB, Kumar P, Oh KS (2009) Comparison of singleand double-bundle anterior cruciate ligament reconstruction using quadriceps tendon-bone autografts. Arthroscopy 25:70-77

6. Mae T, Shino K, Matsumoto N, Maeda A, Nakata K, Yoneda M (2010) Graft tension during active knee extension exercise in anatomic double-bundle anterior cruciate ligament reconstruction. Arthroscopy 26:214-222

7. Mae T, Shino K, Miyama T, Shinjo H, Ochi T, Yoshikawa H, Fujie H (2001) Single- versus two-femoral socket anterior cruciate ligament reconstruction technique: biomechanical analysis using a robotic simulator. Arthroscopy 17:708-716

8. Monaco E, Labianca L, Conteduca F, De Carli A, Ferretti A (2007) Double bundle or single bundle plus extraarticular tenodesis in ACL reconstruction? A CAOS study. Knee Surg Sports Traumatol Arthrosc 15:1168-1174

9. Petersen W, Zantop T (2006) Partial rupture of the anterior cruciate ligament. Arthroscopy 22:1143-1145

10. Radice F, Yanez R, Gutierrez V, Rosales J, Pinedo M, Coda S (2010) Comparison of magnetic resonance imaging findings in anterior cruciate ligament grafts with and without autologous platelet-derived growth factors. Arthroscopy 26:50-57

11. Schenck RC Jr, Kovach IS, Agarwal A, Brummett R, Ward RA, Lanctot D, Athanasiou KA (1999) Cruciate injury patterns in knee hyperextension: a cadaveric model. Arthroscopy 15:489495

12. Schreiber VM, van Eck CF, Fu FH (2010) Anatomic doublebundle ACL reconstruction. Sports Med Arthrosc 18:27-32

13. Shen W, Forsythe B, Ingham SM, Honkamp NJ, Fu FH (2008) Application of the anatomic double-bundle reconstruction concept to revision and augmentation anterior cruciate ligament surgeries. J Bone Joint Surg Am 90 (Suppl 4):20-34

14. Siebold R, Fu FH (2008) Assessment and augmentation of symptomatic anteromedial or posterolateral bundle tears of the anterior cruciate ligament. Arthroscopy 24:1289-1298 
15. Steckel H, Vadala G, Davis D, Fu FH (2006) 2D and 3D 3-tesla magnetic resonance imaging of the double bundle structure in anterior cruciate ligament anatomy. Knee Surg Sports Traumatol Arthrosc 14:1151-1158

16. Tashman S, Collon D, Anderson K, Kolowich P, Anderst W (2004) Abnormal rotational knee motion during running after anterior cruciate ligament reconstruction. Am J Sports Med 32:975-983

17. Toritsuka Y, Amano H, Kuwano M, Iwai T, Mae T, Ohzono K, Shino K (2009) Outcome of double-bundle ACL reconstruction using hamstring tendons. Knee Surg Sports Traumatol Arthrosc $17: 456-463$

18. van Eck CF, Lesniak BP, Schreiber VM, Fu FH (2010) Anatomic single- and double-bundle anterior cruciate ligament reconstruction flowchart. Arthroscopy 26:258-268

19. van Eck CF, Schreiber VM, Liu TT, Fu FH (2010) The anatomic approach to primary, revision and augmentation anterior cruciate ligament reconstruction. Knee Surg Sports Traumatol Arthrosc. doi:10.1007/s00167-010-1191-4

20. Weninger P, Zifko B, Liska M, Spitaler R, Pelinka H, Hertz H (2008) Anterior cruciate ligament reconstruction using autografts and double biodegradable femoral cross-pin fixation: functional, radiographic and MRI outcome after 2-year minimum follow-up. Knee Surg Sports Traumatol Arthrosc 16:988-995
21. Woo SL, Kanamori A, Zeminski J, Yagi M, Papageorgiou C, Fu FH (2002) The effectiveness of reconstruction of the anterior cruciate ligament with hamstrings and patellar tendon. A cadaveric study comparing anterior tibial and rotational loads. J Bone Joint Surg Am 84-A:907-914

22. Yagi M, Kuroda R, Nagamune K, Yoshiya S, Kurosaka M (2007) Double-bundle ACL reconstruction can improve rotational stability. Clin Orthop Relat Res 454:100-107

23. Yagi M, Wong EK, Kanamori A, Debski RE, Fu FH, Woo SL (2002) Biomechanical analysis of an anatomic anterior cruciate ligament reconstruction. Am J Sports Med 30:660-666

24. Yamamoto Y, Hsu WH, Woo SL, Van Scyoc AH, Takakura Y, Debski RE (2004) Knee stability and graft function after anterior cruciate ligament reconstruction: a comparison of a lateral and an anatomical femoral tunnel placement. Am J Sports Med 32:1825-1832

25. Yasuda K, Ichiyama H, Kondo E, Miyatake S, Inoue M, Tanabe Y (2008) An in vivo biomechanical study on the tension-versusknee flexion angle curves of 2 grafts in anatomic double-bundle anterior cruciate ligament reconstruction: effects of initial tension and internal tibial rotation. Arthroscopy 24:276-284

26. Zantop T, Brucker PU, Vidal A, Zelle BA, Fu FH (2007) Intraarticular rupture pattern of the ACL. Clin Orthop Relat Res $454: 48-53$ 